\title{
Experimental Investigation of Co-channel and Adjacent Channel Operations of Microwave Power and IEEE 802.11g Data Transmissions*
}

\author{
Norikatsu IMOTO $^{\dagger \text { a) }}$, Shota YAMASHITA ${ }^{\dagger}$, Student Members, Takuya ICHIHARA ${ }^{\dagger \dagger}$, Nonmember, \\ Koji YAMAMOTO $^{\dagger}$, Senior Member, Takayuki NISHIO ${ }^{\dagger}$, Member, Masahiro MORIKURA $^{\dagger}$, Fellow, \\ and Naoki SHINOHARA ${ }^{\dagger \dagger}$, Member
}

\begin{abstract}
SUMMARY We discuss the division of radio resources in the time and frequency domains for wireless local area network (WLAN) devices powered with microwave energy. In general, there are two ways to avoid microwave power transmission (MPT) from influencing data communications: adjacent channel operation of continuous MPT and WLAN data transmission and co-channel operation of intermittent MPT and WLAN data transmission. Experimental results reveal that, even when we implement these methods, several problems arise because WLAN devices have been developed without supposing the existence of MPT. One problem clarified in our experiment is that adjacent channel operation at $2.4 \mathrm{GHz}$ does not necessarily perform well owing to the interference from MPT. This interference occurs regardless of the frequency separation at $2.4 \mathrm{GHz}$. The other problem is that intermittent MPT could result in throughput degradation owing to the data rate control algorithm and the association scheme of the WLAN. In addition, the experimental results imply that a microwave energy source and a WLAN device should share information on the timings of intermittent MPT and data transmission to avoid buffer overflow. key words: microwave power transmission, IEEE 802.11, CSMA/CA, WLAN, adjacent channel interference
\end{abstract}

\section{Introduction}

With the wide deployment of wireless machine-to-machine networks, exploiting batteryless devices in wireless networks has been desired. Wireless networks powered with batteries suffer from a limited lifetime. In contrast, batteryless devices would free us from battery replacement and disposal: therefore, they have the potential to make wireless networks ubiquitous.

In recent years, much of the previous work on batteryless devices has focused on energy harvesting [1], [2] and wireless powering [3], [4], including radio-frequency (RF) inductive powering [5] and microwave power transmission (MPT) [6]-[10]. In particular, MPT is more efficient at longer range than the use of RF inductive powering and can be controlled more actively than energy-harvesting schemes. By powering wireless devices actively and selectively, we can extend the network lifetime. Because MPT

\footnotetext{
Manuscript received January 6, 2014.

Manuscript revised April 22, 2014.

$\dagger$ The authors are with the Graduate School of Informatics, Kyoto University, Kyoto-shi, 606-8501 Japan.

${ }^{\dagger}$ The authors are with the Research Institute for Sustainable Humanosphere, Kyoto University, Uji-shi, 611-0011 Japan.

*This paper was presented in part at IEEE PIMRC 2013.

a) E-mail: info14@imc.cce.i.kyoto-u.ac.jp

DOI: 10.1587/transcom.E97.B.1835
}

would interfere with data receptions of wireless local area network (WLAN) devices when the frequency of continuous MPT is set to the same channel as that used for WLANs, the microwave power should be transmitted on a separate channel from the data transmissions or should be transmitted intermittently.

There is much research on MPT for wireless devices. In [6], [7], the authors have succeeded in designing antennas and rectifiers. In [8], [9], the authors have succeeded in powering mobile phones or sensor devices wirelessly using the 2.4-GHz band. In these previous works, the effects of MPT on the WLAN communications are not discussed in detail. In [10], the authors have investigated the effects of MPT on IEEE 802.15-based communications.

The main purpose of this paper is to experimentally investigate the impact of MPT on IEEE 802.11g-based WLAN data communications. In particular, we discuss a model where a wireless device is powered wirelessly and the device tries to transmit its data. We would like to note that a problem that MPT to a device could interfere with data transmission of other devices is out of scope of this study. In addition, in particular, we discuss protocol issues such as the division of radio resources in the time and frequency domains. On the other hand, we do not discuss issues related to the amount of supplied power.

The motivation for using IEEE $802.11 \mathrm{~g}$ is as follows. Our big goal is to power IEEE 802.11ah [11] devices wirelessly in the $900-\mathrm{MHz}$ band. IEEE 802.11ah is based on carrier sense multiple access with collision avoidance (CSMA/CA) and is in the standardization phase, and it targets low-rate and low-power-consumption services such as sensor networks. Because there are no devices using the IEEE 802.11ah protocol at present, we use IEEE 802.11gbased WLAN devices in the present study. Note that both IEEE 802.11ah and IEEE 802.11g are CSMA/CA-based standards.

In this study, the microwave power is set to be transmitted at the same 2.4-GHz band toward efficient utilization of the spectrum. There are two means of avoiding interference from MPT to data communications: adjacent channel operation of continuous microwave power and data transmissions and co-channel operation of intermittent microwave power and data transmissions. However, there are several problems 
that cannot be solved by these methods because WLAN devices have been developed without supposing the existence of MPT. Adjacent channel WLAN devices would be affected by MPT because they have been developed without supposing the amount of power from a microwave energy source. On the other hand, in general, WLAN communications can be conducted successfully because of CSMA/CA when microwave power is transmitted intermittently. However, the WLAN devices may fail to receive beacon frames because they have been developed without supposing the length of the period during MPT.

This paper is organized as follows. Section 2 describes adjacent channel operation of continuous MPT and WLAN data transmissions. In Sect. 3, co-channel operation of intermittent MPT and WLAN data transmission is described. Section 4 summarizes the main findings.

\section{Adjacent Channel Operation of Continuous MPT and WLAN Data Transmission}

In this section, we measure the throughput in adjacent channel operation to discuss the feasibility of adjacent channel operation of continuous MPT and IEEE 802.11-based data transmission. In particular, both the impact of received power density at a WLAN device and that of the frequency separation on the throughput are evaluated. Even when using adjacent channel operation, WLAN devices would be affected by the influence of MPT. This is because the received MPT power is quite large and thus cannot be attenuated by using a band-pass filter.

\subsection{Experimental Setup for Continuous MPT}

Figure 1 shows the setup of our experiment. In the experiment, the system consists of an energy source (ES), a data transmitter (DT), and a data receiver (DR). All measurements in Sect. 2 and Sect. 3 are performed in a radioanechoic chamber, i.e., there are no other transmitters. The DT transmits data frames to the DR at a center frequency of $2.457 \mathrm{GHz}$. The ES transmits continuous microwave to the DT. Note that the use of continuous microwave for MPT is a general assumption as in [3], [4], [7], [8]. We have confirmed that the bandwidth for MPT is less than $2 \mathrm{kHz}$ through measurements. In addition, the center frequency, $f_{\text {MPT }}$, is set to be in the band from 2.4 to $2.5 \mathrm{GHz}$. The ES, DT, and DR are placed on a straight line. Figure 2 shows the horn antenna of the ES and the DT used in this experiment. The distance between the ES and the DT is set to be $2.46 \mathrm{~m}$, and the distance between the ES and the DR is set to be $2.49 \mathrm{~m}$. The DR is positioned behind the ES to avoid the influence from MPT. In this experiment, we need only one DT, and the DT is not powered wirelessly. This is because the purpose of this experiment is to discuss the feasibility of adjacent channel operation of continuous MPT and WLAN data transmission, not the feasibility in terms of the amount of supplied power.

The ES consists of a signal generator, an amplifier, and

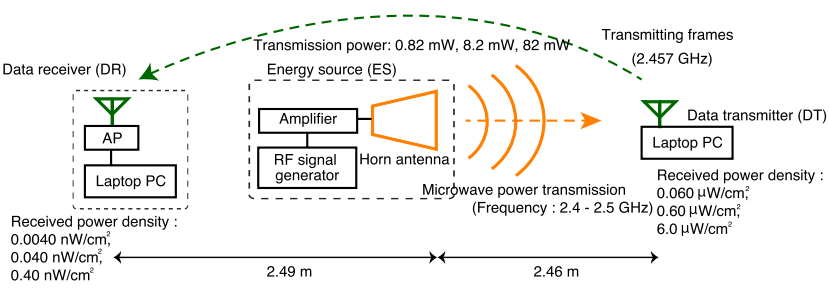

Fig. 1 Setup of adjacent channel operation.

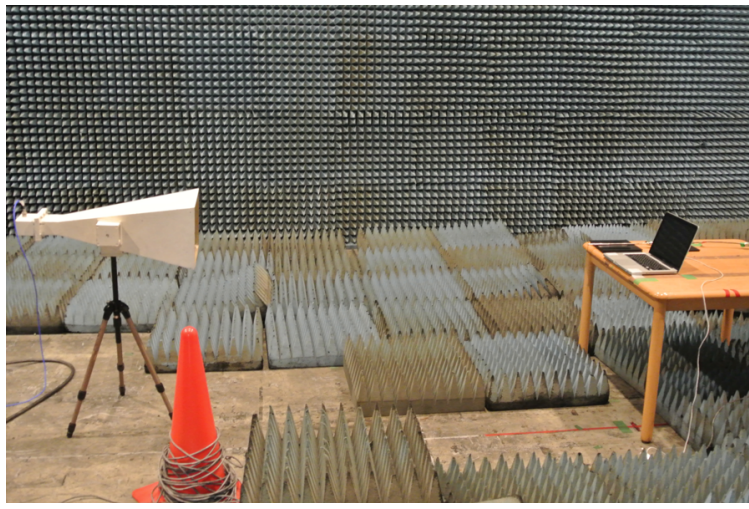

Fig. 2 Experimental setup of the horn antenna and the DT.

a horn antenna. When the transmission power of the ES is set to be $0.82,8.2$, and $82 \mathrm{~mW}$, the received power density at the DT is $0.060,0.60$, and $6.0 \mu \mathrm{W} / \mathrm{cm}^{2}$, respectively.

We use a laptop PC (Apple MacBook Pro) as a DT. The DT transmits User Datagram Protocol (UDP) data frames using Iperf 2.0.5 [12] for $20 \mathrm{~s}$. The offered load is set to be $15 \mathrm{Mbit} / \mathrm{s}$, and the UDP datagram size is set to be $1470 \mathrm{~B}$.

The DR consists of an access point (Allied Telesis ATTQ2403) and a laptop PC. Using Iperf, the laptop PC on the $\mathrm{DR}$ receives data frames from the DT via the access point (AP) and measures the throughput.

\subsection{Adjacent Channel Operation Results}

Figure 3 shows the results of the experiments. The throughput characteristics highly depend on the received power density at the DT. When the received power density at the DT was $\leq 0.60 \mu \mathrm{W} / \mathrm{cm}^{2}$, a throughput of approximately $15 \mathrm{Mbit} / \mathrm{s}$ was achieved unless the center frequency of the MPT, $f_{\mathrm{MPT}}$, overlapped the channel for the WLAN. Here, recall that the offered load is $15 \mathrm{Mbit} / \mathrm{s}$. The reason the DT transmitted data frames at $15 \mathrm{Mbit} / \mathrm{s}$ is that it did not detect microwave energy from the ES. In contrast, the DT detected microwave energy from the ES when $f_{\mathrm{MPT}}$ overlapped the channel for WLAN communication; thus, the throughput was essentially zero.

On the other hand, when the received power density at the DT was $6.0 \mu \mathrm{W} / \mathrm{cm}^{2}$, the DT detected microwave energy from the ES regardless of $f_{\mathrm{MPT}}$ because the band-pass filter at the receiver does not completely attenuate the microwave energy.

If WLAN devices are powered wirelessly, the received 




Received power density of DT $0.060 \mu \mathrm{W} / \mathrm{cm}^{2}$ $0.60 \mu \mathrm{W} / \mathrm{cm}^{2}$ $6.0 \mu \mathrm{W} / \mathrm{cm}^{2}$

Fig. 3 Throughput of adjacent channel operation.

power density at the devices generally needs to be much greater than $6.0 \mu \mathrm{W} / \mathrm{cm}^{2}$. In this case, the WLAN modules would detect microwave energy from the ES regardless of the value of $f_{\mathrm{MPT}}$. For example, as estimated in [13], the WLAN sensor node needs to receive a power density of $0.3 \mathrm{~mW} / \mathrm{cm}^{2}$, even in sleep mode. There are at least two possible solutions. The first solution is to use an appropriate band-pass filter at the receiver to attenuate microwave power of MPT. The second solution is to transmit microwave power intermittently. Since the second solution can be implemented by using commercially available devices, in this paper, we discuss only on intermittent MPT. In this case, co-channel operation of MPT and data transmission is more appropriate than adjacent channel operation in terms of the available bandwidth for other systems using the 2.4-GHz band.

\section{Co-Channel Operation of Intermittent MPT and WLAN Data Transmissions}

In this section, the feasibility of the co-channel operation of intermittent MPT and WLAN data transmission is discussed. Because WLAN devices are operated on the basis of CSMA/CA, WLAN devices seem to successfully communicate even when microwave power is transmitted intermittently. However, the effect of other control schemes including the association scheme is of concern.

To discuss the feasibility in detail, we measure the data rate and the number of discarded data frames. In addition, we discuss unknown issues caused by application of MPT to WLAN devices via comparison with experimental data.

\subsection{Experimental Setup for Intermittent MPT}

Figure 4 shows the setup of the experiment, which consists of an ES, a DT, a DR, and a frame analyzer (FA). The ES, DT, and DR are placed on a straight line. In addition, the DR and FA are positioned behind the ES to avoid the influence from MPT. As in the previous experiment in Sect. 2,

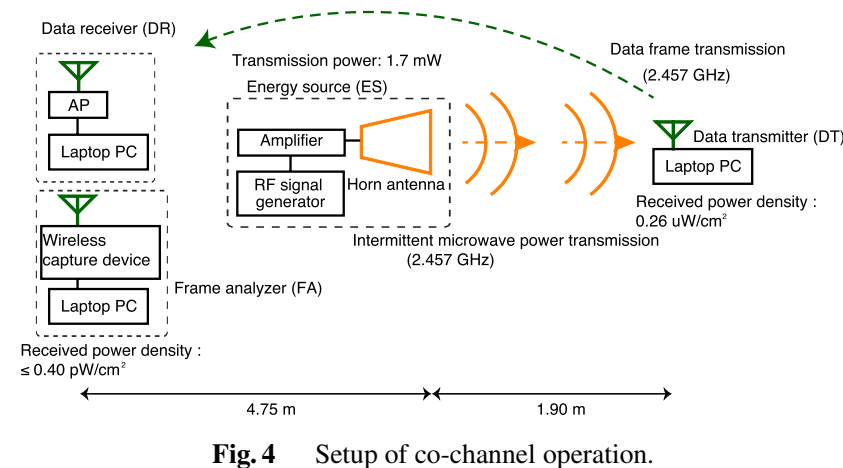

we need only one DT in this experiment, and the DT is not powered wirelessly. This is because the purpose of this experiment is to discuss the feasibility of co-channel operation of intermittent MPT and WLAN data transmission, not the feasibility in terms of the amount of supplied power.

The FA consists of a WLAN capture device and a laptop PC and captures the frames from the DT and DR. The motivation for conducting frame capturing is to investigate the data rate that is specified in the header of each frame and the number of transmitted frames in detail. Note that in the experiment in Sect. 3, we have no need to use the FA because the purpose of the experiment was to investigate whether the DT detects microwave energy or not.

The ES transmits microwave power intermittently; specifically, it transmits microwave power during a fixed time, $T_{\mathrm{PT}}$, and stops MPT during the other fixed time, $T_{\mathrm{PS}}$, periodically. Note that the subscript "PT" represents "power transmission," and the subscript "PS" represents "power suspension." For the FA to correctly capture data frames from the DT even if the ES transmits microwave power, we change the positions of devices and the transmission power of the ES from those in Sect. 2. The ES is set to transmit microwave power intermittently at a center frequency of $2.457 \mathrm{GHz}$ and a transmission power of $1.70 \mathrm{~mW}$. Moreover, the distance between the ES and the DT is set to be $1.90 \mathrm{~m}$, and the distance between the ES and the DR is set to be $4.75 \mathrm{~m}$. Note that this power setting does not affect the operation of the DT and DR.

During $T_{\mathrm{PT}}$, the DT detects microwave energy from the ES; hence, the DT seems not to transmit data frames and holds them in a finite-size buffer during $T_{\mathrm{PT}}$, and the DT would transmit them in $T_{\mathrm{PS}}$. As in the previous experiment in Sect. 2, the offered load is set to be $15 \mathrm{Mbit} / \mathrm{s}$ in this experiment, and the UDP datagram size is set to be $1470 \mathrm{~B}$.

\subsection{Theoretical Estimation of Frame Loss Rate}

In general, a WLAN module employing CSMA/CA seems to transmit data frames successfully when microwave power is transmitted intermittently. This is because the WLAN module detects the microwave energy and avoids collisions between data frames and microwave power. However, the DT appears to delete particular frames in the buffer, if the output buffer is full, according to a pre-programmed buffer 
management rule [14]. Thus, in the experiments, the buffer management rule seems to have considerable influence on the number of discarded frames. In this section, for simplicity, we discuss the frame loss caused by buffer overflow with tail-drop.

The frame loss rate $P_{\text {loss }}$ is defined as

$$
P_{\text {loss }}:=\frac{N_{\text {generated }}-N_{\text {received }}}{N_{\text {generated }}},
$$

where $N_{\text {received }}$ is the number of received frames, and $N_{\text {generated }}$ is the number of generated frames. In particular, we discuss the impact of $T_{\mathrm{PT}}$ and $T_{\mathrm{PS}}$ on the frame loss rate.

The output buffer on the DT seems to overflow mainly when $T_{\mathrm{PT}}$ is long, or $T_{\mathrm{PS}}$ is short. This is because more data frames will be stored in the buffer during $T_{\mathrm{PT}}$ when $T_{\mathrm{PT}}$ is larger. In addition, fewer data frames can be transmitted during $T_{\mathrm{PS}}$ when $T_{\mathrm{PT}}$ is smaller; as a result, the buffer is likely to be full. To confirm these hypotheses and estimate the conditions to prevent frame loss, we formulate the frame loss rate as a function of $T_{\mathrm{PT}}$ and $T_{\mathrm{PS}}$. For the sake of simplicity, we assume that the DT does not transmit data frames during $T_{\mathrm{PT}}$.

First, when $T_{\mathrm{PS}}$ is long enough, whether or not the output buffer overflows depends on the value of $T_{\mathrm{PT}}$. Intuitively, it seems clear that the output buffer overflows when the sum of the size of the data frames generated during $T_{\mathrm{PT}}$ is larger than the output buffer size $Z$. Therefore, the condition to prevent buffer overflow is:

$$
G T_{\mathrm{PT}} \leq Z,
$$

where $G$ is an offered load. Here, the equality of (2) holds if

$$
T_{\mathrm{PT}}=Z / G=: T_{\mathrm{PT}, \text { longPS }} .
$$

Therefore, when $T_{\mathrm{PS}}$ is long enough and $T_{\mathrm{PT}}>T_{\mathrm{PT}, \text { longPS }}$, the number of discarded frames, $N_{\text {generated }}-N_{\text {received }}=$ : $N_{\text {discarded }}$, is calculated as follows:

$$
N_{\text {discarded }}=\frac{G T_{\mathrm{PT}}-Z}{L},
$$

where $L$ is the UDP payload size.

Second, when $T_{\mathrm{PT}} \leq T_{\mathrm{PT}, \text { longPS }}$, whether or not the output buffer overflows depends on the ratio of $T_{\mathrm{PS}}$ to $T_{\mathrm{PT}}$. When the number of data frames generated in a cycle is larger than the number of data frames that can be transmitted in $T_{\mathrm{PS}}$, the buffer would overflow. Therefore, the condition to prevent buffer overflow is:

$$
\frac{G\left(T_{\mathrm{PT}}+T_{\mathrm{PS}}\right)}{L} \leq \frac{T_{\mathrm{PS}}}{\tau},
$$

where $\tau$ represents the average value of the periods from the start of data transmission to the start of the next data transmission. Note that the value of $\tau$ is evaluated experimentally. Here, the equality of (5) holds if

$$
T_{\mathrm{PS}}=\frac{G T_{\mathrm{PT}}}{L / \tau-G}=: T_{\mathrm{PS}, \text { shortPT }} .
$$

Therefore, when $T_{\mathrm{PT}} \leq T_{\mathrm{PT} \text {,longPS }}$ and $T_{\mathrm{PS}}<T_{\mathrm{PS} \text {,shortPT }}$, $N_{\text {discarded }}$ is calculated as follows:

$$
N_{\text {discarded }}=\frac{G\left(T_{\mathrm{PT}}+T_{\mathrm{PS}}\right)-L T_{\mathrm{PS}} / \tau}{L} .
$$

Third, when $T_{\mathrm{PT}}>T_{\mathrm{PT}, \text { longPS }}$, whether $N_{\text {discarded }}$ is calculated by (4) or (7) depends on the value of $T_{\mathrm{PS}}$. In this case, the DT should transmit $\left(Z+G T_{\mathrm{PS}}\right) / L$ of data frames during $T_{\mathrm{PS}}$. Therefore, when

$$
\frac{Z+G T_{\mathrm{PS}}}{L} \leq \frac{T_{\mathrm{PS}}}{\tau},
$$

$N_{\text {discarded }}$ is calculated by (7). Here, the equality of (8) holds if

$$
T_{\mathrm{PS}}=\frac{Z}{L / \tau-G}=: T_{\mathrm{PS}, \text { longPT }} .
$$

Consequently, by using equations from (2)-(6) and $N_{\text {generated }}=G\left(T_{\mathrm{PT}}+T_{\mathrm{PS}}\right) / L$, the frame loss rate $P_{\text {loss }}$ can be calculated as

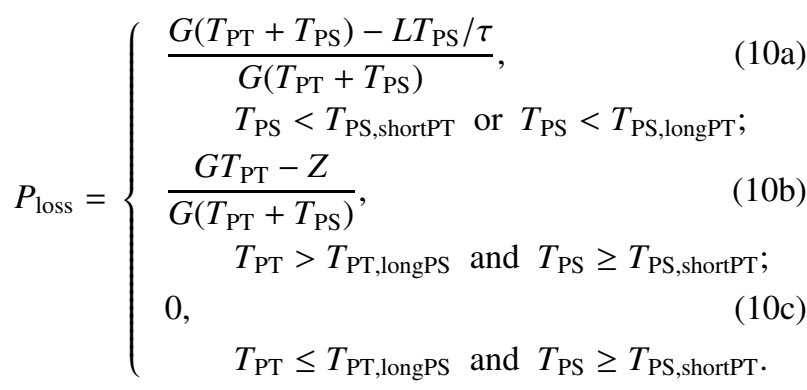

We would like to emphasize that $P_{\text {loss }}$ is an increasing function of $T_{\mathrm{PT}}$, and it is a decreasing function of $T_{\mathrm{PS}}$.

To avoid buffer overflow, $T_{\mathrm{PT}} \leq T_{\mathrm{PT} \text {,longPS }}$ and $T_{\mathrm{PS}} \geq$ $T_{\mathrm{PS} \text {,shortPT }}$ are required. Thus, the ratio of $T_{\mathrm{PS}}$ to $T_{\mathrm{PT}}$ should satisfy:

$$
\begin{aligned}
T_{\mathrm{PS}} / T_{\mathrm{PT}} & \geq T_{\mathrm{PS}, \text { shortPT }} / T_{\mathrm{PT}, \text { longPS }} \\
& =\frac{1}{L / G \tau-1} ;
\end{aligned}
$$

i.e., the lower bound of $T_{\mathrm{PS}} / T_{\mathrm{PT}}$ needs to be increased along with the offered load $G$. Note that a larger value of $T_{\mathrm{PS}} / T_{\mathrm{PT}}$ means more time for data transmission and less time for MPT.

The existence of the lower bound for $T_{\mathrm{PS}} / T_{\mathrm{PT}}$ means that there is an upper bound for the supplied power. We let the supplied power during $T_{\mathrm{PT}}$ be denoted by $P_{\mathrm{PT}}$. Then, the average supplied power over multiple cycles, $P_{\mathrm{e}}$, can be written as

$$
\begin{aligned}
P_{\mathrm{e}} & =\frac{P_{\mathrm{PT}} T_{\mathrm{PT}}}{T_{\mathrm{PT}}+T_{\mathrm{PS}}}=\frac{P_{\mathrm{PT}}}{1+T_{\mathrm{PS}} / T_{\mathrm{PT}}} \\
& \leq \frac{P_{\mathrm{PT}}}{1+T_{\mathrm{PS}, \text { shortPT }} / T_{\mathrm{PT}, \text { longPS }}} \\
& =(1-G \tau / L) P_{\mathrm{PT}}=: P_{\mathrm{e}, \text { max }} .
\end{aligned}
$$

Thus, the larger offered traffic $G$ means a lower average supplied power. Figure 5 shows the relation between $P_{\mathrm{e}, \max } / P_{\mathrm{PT}}$ 




Fig. $5 \quad P_{\mathrm{e}, \max } / P_{\mathrm{PT}}$ versus offered load $G$ for $\tau=0.67 \mathrm{~ms}$ and $L=1470 \mathrm{~B}$.

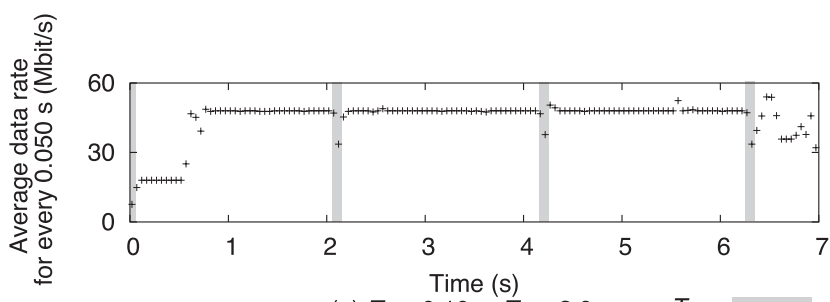

(a) $T_{\mathrm{PT}}=0.10 \mathrm{~s}, T_{\mathrm{PS}}=2.0 \mathrm{~s}$. $\quad T_{\mathrm{PT}}$

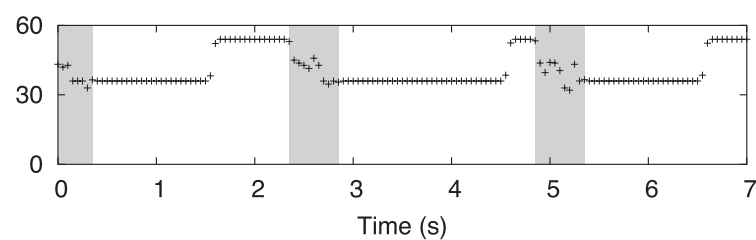

(b) $T_{\mathrm{PT}}=0.50 \mathrm{~s}, T_{\mathrm{PS}}=2.0 \mathrm{~s} . \quad T_{\mathrm{PT}}$

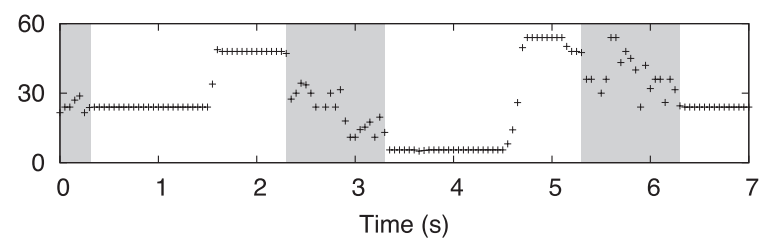

(c) $T_{\mathrm{PT}}=1.0 \mathrm{~s}, T_{\mathrm{PS}}=2.0 \mathrm{~s} . \quad T_{\mathrm{PT}}$

Fig. 6 Average data rate for every $0.050 \mathrm{~s}$ for $T_{\mathrm{PT}}=0.10 \mathrm{~s}$ and $T_{\mathrm{PS}}=$ $2.0 \mathrm{~s}$, for $T_{\mathrm{PT}}=0.50 \mathrm{~s}$ and $T_{\mathrm{PS}}=2.0 \mathrm{~s}$, and for $T_{\mathrm{PT}}=1.0 \mathrm{~s}$ and $T_{\mathrm{PS}}=2.0 \mathrm{~s}$.

and $G$ for $\tau=0.67 \mathrm{~ms}$ and $L=1470 \mathrm{~B}$. $P_{\mathrm{e}, \max } / P_{\mathrm{PT}}$ decreases as $G$ increases. Therefore, $G$ needs to decrease to supply enough power to a WLAN device.

\subsection{Co-Channel Operation Results}

\subsubsection{Fluctuations in Data Rate Because of MPT}

Figures 6(a), 6(b), and 6(c) show the average data rate for every $0.050 \mathrm{~s}$ for $T_{\mathrm{PT}}=0.10 \mathrm{~s}$ and $T_{\mathrm{PS}}=2.0 \mathrm{~s}$, for $T_{\mathrm{PT}}=$ $0.50 \mathrm{~s}$ and $T_{\mathrm{PS}}=2.0 \mathrm{~s}$, and for $T_{\mathrm{PT}}=1.0 \mathrm{~s}$ and $T_{\mathrm{PS}}=2.0 \mathrm{~s}$, respectively. We can see that the data rate was decreased during $T_{\mathrm{PT}}$.

In detail, we can see that there are two factors in Fig. 6 that we have not taken into account in Sect. 3.2. These fac-

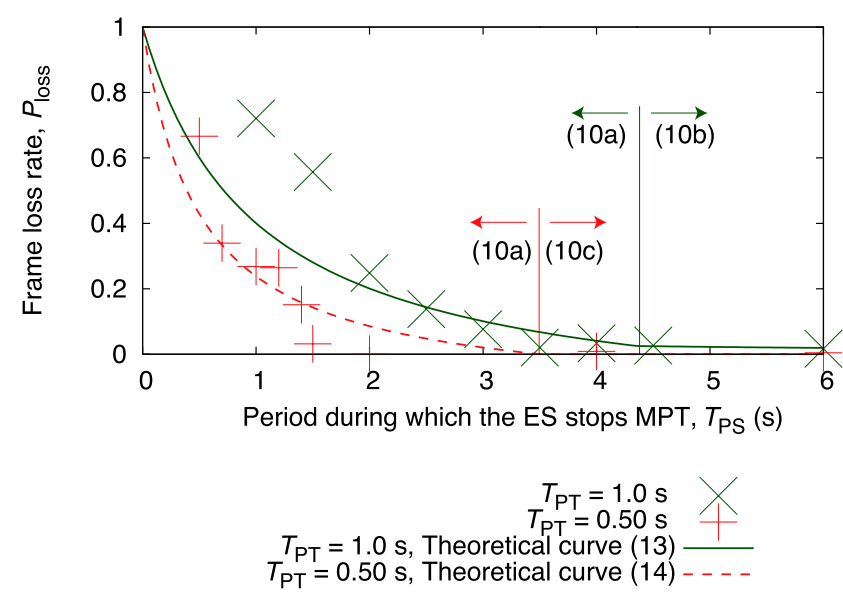

Fig. 7 Frame loss rate versus period during which the ES suspends MPT. The solid line represents the fitting of the experimental data of $T_{\mathrm{PT}}=1.0 \mathrm{~s}$ to theoretical estimations of frame loss rate provided $Z=1.6 \mathrm{MB}$ and $\tau=$ $0.65 \mathrm{~ms}$. The dashed line represents the fitting of the experimental data of $T_{\mathrm{PT}}=0.5 \mathrm{~s}$ to theoretical estimations of frame loss rate provided $Z=$ $1.6 \mathrm{MB}$ and $\tau=0.68 \mathrm{~ms}$.

tors are related to data rate control, and they increase the frame loss rate more than that estimated in (10a), (10b), and (10c). The first factor is that the DT attempts to retransmit data frames at a decreased data rate during $T_{\mathrm{PT}}$ as shown in Fig. 6. This is because the DT sometimes attempts to transmit and fail to receive an acknowledgment (ACK) frame, even during $T_{\mathrm{PT}}$. In particular, the data rate gradually decreased along with $T_{\mathrm{PT}}$. Note that the data rate control is not standardized and depends on the device characteristics. The second factor is that a certain amount of time is required for the DT to reconfigure the data rate after the ES stops MPT as can be seen in Figs. 6(b) and 6(c).

As shown in Fig. 6(a), the DT generally transmits data frames at $48 \mathrm{Mbit} / \mathrm{s}$ during $T_{\mathrm{PS}}$. Note that when we set $T_{\mathrm{PT}}<0.10 \mathrm{~s}$, similar trends to the case of $T_{\mathrm{PT}}=0.10 \mathrm{~s}$ would be achieved. On the other hand, when $T_{\mathrm{PT}}$ is long enough and $T_{\mathrm{PS}}$ is shorter than the time required for the data rate of $48 \mathrm{Mbit} / \mathrm{s}$, the DT would not reconfigure the data rate even after the ES stops MPT.

If the DT is controlled not to transmit data frames during MPT, the DT is expected not to decrease its data rate. The ES and the DT need to share information on the timings of MPT and data transmission to enable this control.

\subsubsection{Frame Loss Rate}

Figure 7 shows the frame loss rate versus $T_{\mathrm{PS}}$ for $T_{\mathrm{PT}}=$ $0.50 \mathrm{~s}$ and $T_{\mathrm{PT}}=1.0 \mathrm{~s}$. Here, $T_{\mathrm{PS}}$ is set to be $\geq 0.50 \mathrm{~s}$ in the experiment for $T_{\mathrm{PT}}=1.0 \mathrm{~s}$. This is because similar trends to the case of $T_{\mathrm{PS}}=0.50 \mathrm{~s}$ would be achieved as long as $T_{\mathrm{PS}}$ is shorter than the time required to reconfigure the data rate to $48 \mathrm{Mbit} / \mathrm{s}$. In addition, $T_{\mathrm{PS}}$ is set to be $\geq 0.50 \mathrm{~s}$ also in the experiment for $T_{\mathrm{PT}}=0.50 \mathrm{~s}$. This is because the purpose of our experiments is to reveal unknown issues as data rate degradation. Note that when $T_{\mathrm{PT}}<0.10 \mathrm{~s}$, the data rate would not be reconfigured during $T_{\mathrm{PS}}$. The frame loss rate 
$P_{\text {loss }}$ decreased as $T_{\mathrm{PS}}$ increased, as can be easily understood from (10a), (10b), and (10c). We can see that (10a) and (10b) are appropriate models for the relation between $P_{\text {loss }}$ and $T_{\mathrm{PS}}$ when $T_{\mathrm{PT}}=1.0 \mathrm{~s}$ in the range $T_{\mathrm{PS}} \geq 2.0 \mathrm{~s}$. In this range, $\tau=0.65 \mathrm{~ms}$ is measured independent of $T_{\mathrm{PS}}$, and $Z$ is estimated by fitting the experimental data $T_{\mathrm{PT}}=1.0 \mathrm{~s}$ in this range to the theoretical estimations (10a), (10b), and (10c) using a least-squares method. Therefore, the theoretical estimations of the frame loss rate are calculated as follows:

$$
P_{\text {loss }}= \begin{cases}\frac{1.2 \mathrm{~s}}{T_{\mathrm{PS}}+1.0 \mathrm{~s}}-0.21, & T_{\mathrm{PS}}<4.4 \mathrm{~s} ; \\ \frac{0.15 \mathrm{~s}}{T_{\mathrm{PS}}+1.0 \mathrm{~s}}, & T_{\mathrm{PS}} \geq 4.4 \mathrm{~s} .\end{cases}
$$

Note that $T_{\mathrm{PS} \text {,longPT }}$ is calculated as $4.4 \mathrm{~s}$ using (9). In the other range, i.e., when $T_{\mathrm{PT}}=1.0 \mathrm{~s}$ and $T_{\mathrm{PS}} \leq 1.5 \mathrm{~s}$, the theoretical estimations do not fit the experimental data well. This is caused by the first and second factors that we have not taken into account in Sect. 3.2. In particular, the second factor is the main cause. Recall that, when $T_{\mathrm{PS}}$ is short, the period during which the DT transmits at a low rate becomes longer.

We can see that (10a) and (10c) are appropriate models for the relation between $P_{\text {loss }}$ and $T_{\mathrm{PS}}$ when $T_{\mathrm{PT}}=0.50 \mathrm{~s}$ in the range $T_{\mathrm{PS}} \geq 0.70 \mathrm{~s}$. In this range, $\tau=0.69 \mathrm{~ms}$ is measured independent of $T_{\mathrm{PS}}$. Therefore, the theoretical estimation of the frame loss rate is calculated as follows:

$$
P_{\text {loss }}= \begin{cases}\frac{0.58 \mathrm{~s}}{T_{\mathrm{PS}}+0.50 \mathrm{~s}}-0.15, & T_{\mathrm{PS}}<3.5 \mathrm{~s} \\ 0, & T_{\mathrm{PS}} \geq 3.5 \mathrm{~s} .\end{cases}
$$

Note that $T_{\mathrm{PS} \text {,shortPT }}$ is calculated as $3.5 \mathrm{~s}$ using (6). In the other range, i.e., when $T_{\mathrm{PT}}=0.50 \mathrm{~s}$ and $T_{\mathrm{PS}}=0.50 \mathrm{~s}$, the theoretical estimations do not fit the experimental data well. This is also caused by the second factor that we have not taken into account in Sect. 3.2.

Note that the difference between the experimental data for $T_{\mathrm{PT}}=1.0 \mathrm{~s}$ and the theoretical curve (13) is larger than that between the experimental data for $T_{\mathrm{PT}}=0.50 \mathrm{~s}$ and the theoretical curve (14). This is mainly caused by the first factor that we have not taken into account in Sect. 3.2. Recall that the longer $T_{\mathrm{PT}}$ is, the more the DT fails to receive ACK frames, and thus, the data rate decreases.

Figure 8 shows the frame loss rate $P_{\text {loss }}$ versus $T_{\mathrm{PT}}$ for $T_{\mathrm{PS}}=2.0 \mathrm{~s}$ and $T_{\mathrm{PS}}=6.0 \mathrm{~s}$. Here, $T_{\mathrm{PT}}$ is set to be $\geq 0.10 \mathrm{~s}$ in this experiment. This is because similar trends to the case of $T_{\mathrm{PT}}=0.10 \mathrm{~s}$ would be achieved when $T_{\mathrm{PT}}<0.10 \mathrm{~s}$. The frame loss rate $P_{\text {loss }}$ increases along with $T_{\mathrm{PT}}$, as can be easily understood from (10a), (10b), and (10c). When $T_{\mathrm{PS}}=2.0 \mathrm{~s}$, the theoretical estimations (10a), (10b), and (10c) do not fit the experimental data. This is caused by the second factor mentioned previously. Recall that $T_{\mathrm{PS}}$ is so short that the data rate cannot be reconfigured during $T_{\mathrm{PS}}$.

In addition, we can see that when $T_{\mathrm{PS}}=6.0 \mathrm{~s}$ in the range $0 \leq T_{\mathrm{PT}} \leq 2.5 \mathrm{~s}$, (10b) and (10c) are appropriate models for the relation between $P_{\text {loss }}$ and $T_{\mathrm{PT}}$. In this range,



$$
\begin{array}{r}
T_{\mathrm{PS}}=6.0 \mathrm{~s} \\
T_{\mathrm{PS}}=2.0 \mathrm{~s} \\
T_{\mathrm{PS}}=6.0 \mathrm{~s} \text {, Theoretical curve (15) }
\end{array}
$$

Fig. 8 Frame loss rate versus power transmission period. The solid line represents the fitting of the experimental data of $T_{\mathrm{PS}}=6.0 \mathrm{~s}$ to theoretical estimations of frame loss rate provided $Z=1.9 \mathrm{MB}$ and $\tau=0.67 \mathrm{~ms}$.

$\tau=0.67 \mathrm{~ms}$ is measured independent of $T_{\mathrm{PT}}$, and $Z$ is estimated by fitting the experimental data in the range from $1.0 \mathrm{~s} \leq T_{\mathrm{PT}} \leq 2.5 \mathrm{~s}$ to the theoretical estimation (10b) using a least-squares method. Therefore, when $T_{\mathrm{PS}}=6.0 \mathrm{~s}$, the theoretical estimations of frame loss rate (10b) and (10c) are calculated as follows:

$$
P_{\text {loss }}= \begin{cases}1.0-\frac{5.0 \mathrm{~s}}{T_{\mathrm{PT}}+6.0 \mathrm{~s}}, & T_{\mathrm{PT}}>1.0 \mathrm{~s} \\ 0, & T_{\mathrm{PT}} \leq 1.0 \mathrm{~s} .\end{cases}
$$

Note that $T_{\mathrm{PT} \text {,longPS }}$ is calculated as $1.0 \mathrm{~s}$ using (3).

In the other range, i.e., $T_{\mathrm{PT}} \geq 2.6 \mathrm{~s}$ and $T_{\mathrm{PS}}=6.0 \mathrm{~s}$, the theoretical estimations (10a), (10b), and (10c) do not fit the experimental data well. This is caused by a factor that is described neither in Sect. 3.2 nor in Sect. 3.3.1. Hereafter, we call it the third factor, where the DT often fails to receive frames from the DR during $T_{\mathrm{PT}}$, including beacon frames. We find that the DT attempts to go to sleep and does not transmit data for a certain time when $T_{\mathrm{PT}} \geq 2.6 \mathrm{~s}$. Thus, $P_{\text {loss }}$ is higher than the theoretical estimation. Note that details of sleep control is not standardized and depends on the device characteristics. In addition to these sleep periods, a network disassociation would be caused as the result of beacon reception failure.

To avoid these sleep periods and the network disassociation, for example, microwave power should not be transmitted during a beacon transmission or some timer values concerned with beacon reception should be tuned.

From these results, it has been demonstrated that the theoretical estimations in Sect. 3.2 match the experimental data well, except for the rage in which frame loss rate is affected by any one of the three factors. Owing to these factors, more data frames are discarded than expected in Sect. 3.2.

\section{Conclusion}

We have experimentally clarified the requirements for both 
MPT and IEEE 802.11-based WLAN data transmissions using the same $2.4-\mathrm{GHz}$ band. In particular, we have pointed out three specific issues for MPT that affect WLAN devices. In general, adjacent channel operation of microwave power and data transmissions may be a possible solution. However, we have first demonstrated that almost all data communications failed regardless of the frequency separation in the 2.4$\mathrm{GHz}$ band when the supplied microwave power is enough for the WLAN devices. This may be because the band-pass filter at the receiver does not completely attenuate the microwave energy. Because a great number of WLAN devices have already been used, it is difficult to solve this problem by only changing the frequency of MPT at $2.4 \mathrm{GHz}$.

Thus, we have measured the frame loss rate of data transmissions during intermittent MPT. In general, as a result of CSMA/CA, frames are not discarded by setting the offered load so that the output buffer of the WLAN devices does not overflow. However, we have also found that WLAN devices inefficiently decrease their data rate if they attempt to transmit during MPT. In addition, we have demonstrated that if a WLAN device does not receive consecutive beacons above a certain number owing to MPT, it switches its mode to sleep, or it is disassociated from the AP.

There are many possible solutions to resolve these issues. To avoid the data rate degradation, following solutions seem to be effective: setting the DT not to transmit data frames during MPT, setting the DT not to reduce the data rate, and setting the DT to increase the data rate immediately after MPT is stopped. In addition, to avoid sleep periods and disassociation from the AP, following solutions seem to be effective, i.e., tuning parameters related to time to sleep or time to disassociation and setting the ES not to transmit microwave power while the DT receives beacon frames. In particular, to avoid interference from MPT to beacon receptions, for example, the ES and the DT are required to share information on timings of MPT and data transmission. In contrast, to prevent the transmission of data frames during MPT, for example, sharing information on the timings of MPT and data transmission is required.

Note that some results presented in this paper seem to be specific to WLAN devices used in this study (e.g., data rate control algorithms, adjacent channel rejection, and so forth). However, we would like to emphasize that the purpose of this paper is to reveal unknown issues that are caused by the application of MPT to WLAN devices. In addition, these results at $2.4-\mathrm{GHz}$ band cannot be directly used for IEEE 802.11ah, however, we hope the results presented here will provide useful insights in the standardization process or practical use of IEEE 802.11ah-based devises because similar issues would occur if IEEE 802.11 ah is standardized and developed without considering the existence of MPT.

\section{Acknowledgments}

This work was supported in part by JSPS KAKENHI Grant Number 24360149. This experiment was performed us- ing the Microwave Energy Transmission Laboratory (METLAB) system of the Research Institute for Sustainable $\mathrm{Hu}$ manosphere at Kyoto University.

\section{References}

[1] V. Sharma, U. Mukherji, V. Joseph, and S. Gupta, "Optimal energy management policies for energy harvesting sensor nodes," IEEE Trans. Wireless Commun., vol.9, no.4, pp.1326-1336, April 2010.

[2] K. Tutuncuoglu and A. Yener, "Optimum transmission policies for battery limited energy harvesting nodes," IEEE Trans. Wireless Commun., vol.11, no.3, pp.1180-1189, March 2012.

[3] W.C. Brown, "The history of power transmission by radio waves," IEEE Trans. Microw. Theory Tech., vol.32, no.9, pp.1230-1242, Sept. 1984

[4] T. Paing, J. Morroni, A. Dolgov, J. Shin, J. Brannan, R. Zane, and Z. Popovic, "Wirelessly-powered wireless sensor platform," Proc. European Microwave Conf. 2007, pp.241-244, Munich, Germany, Oct. 2007.

[5] T. Imura, H. Okabe, and Y. Hori, "Basic experimental study on helical antennas of wireless power transfer for electric vehicles by using magnetic resonant couplings," Proc. IEEE Vehicle Power and Propulsion Conf. 2009, pp.936-940, Dearborn, MI, Sept. 2009.

[6] T. Umeda, H. Yoshida, S. Sekine, Y. Fujita, T. Suzuki, and S. Otaka, "A 950-MHz rectifier circuit for sensor network tags with 10-m distance," IEEE J. Solid-State Circuits, vol.41, no.1, pp.35-41, Jan. 2006.

[7] S. Yoshida, T. Noji, G. Fukuda, Y. Kobayashi, and S. Kawasaki, "Experimental demonstration of coexistence of microwave wireless communication and power transfer technologies for battery-free sensor network systems," Int. J. Anntenas and Propagation, pp.1-10, Sept. 2013.

[8] N. Shinohara, M. Tomohiko, and H. Matsumoto, "Study on ubiquitous power source with microwave power transmission," Proc. Union Radio Science (URSI) General Assembly 2005, pp.1-4, 2005

[9] K.M. Farinholt, G. Park, and C.R. Farrar, "RF energy transmission for a low-power wireless impedance sensor node," IEEE J. Sensors Council, vol.9, no.7, pp.793-800, July 2009.

[10] T. Ichihara, T. Mitani, and N. Shinohara, "Study on intermittent microwave power transmission to a ZigBee device," Proc. IEEE Microwave Workshop Series (IMWS) on Innovative Wireless Power Transmission: Technologies, Systems, and Applications 2012, pp.209-212, Kyoto, Japan, May 2012.

[11] IEEE 802.11 Task Group ah (TGah) Sub $1 \mathrm{GHz}$ license-exempt operation, http://www.ieee802.org/11/Reports/tgah_update.htm

[12] Iperf, http://www.iperf.fr

[13] S. Yamashita, N. Imoto, T. Ichihara, K. Yamamoto, T. Nishio, M. Morikura, and N. Shinohara, "Implementation and feasibility study of co-channel operation system of microwave power transmissions to IEEE 802.11-based batteryless sensors," IEICE Trans. Commun., vol.E97-B, no.9, pp.1843-1852, Sept. 2014.

[14] N. Shacham and P.E. McKenney, "Packet recovery in high-speed networks using coding and buffer management," Proc. IEEE INFOCOM'90, vol.1, pp.124-131, San Francisco, CA, June 1990. 


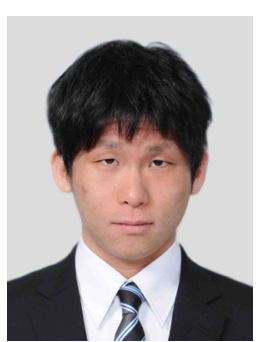

Norikatsu Imoto received his B.E. degree in electrical and electronic engineering from Kyoto University in 2012. He is currently studying toward his M.E. degree at the Graduate School of Informatics, Kyoto University. His research interests concentrate on microwave power transmission for WLAN devices.



Shota Yamashita received his B.E. degree in electrical and electronic engineering from Kyoto University in 2013. He is currently studying toward his M.E. degree at the Graduate School of Informatics, Kyoto University. His research interests concentrate on microwave power transmission for WLAN devices.

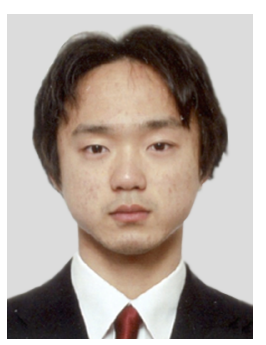

Takuya Ichihara received his B.E. degree in electrical and electronic engineering from Kyoto University in 2012. Since 2012, he has a master's degree student in electrical engineering at Kyoto University. He has been engaged in the study on intermittent microwave power transmission systems.



Koji Yamamoto received the B.E. degree in electrical and electronic engineering from Kyoto University in 2002, and the M.E. and Ph.D. degrees in informatics from Kyoto University in 2004 and 2005, respectively. From 2004 to 2005 , he was a research fellow of the Japan Society for the Promotion of Science (JSPS). Since 2005, he has been with the Graduate School of Informatics, Kyoto University, where he is currently an associate professor. From 2008 to 2009 , he was a visiting researcher at Wireless@KTH, Royal Institute of Technology (KTH) in Sweden. His research interests include the application of game theory, spectrum sharing, and M2M networks. He received the PIMRC 2004 Best Student Paper Award in 2004, the Ericsson Young Scientist Award in 2006, and the Young Researcher's Award from the IEICE of Japan in 2008. He is a member of the IEEE.

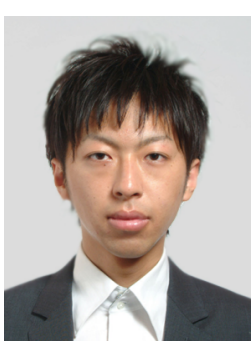

Takayuki Nishio received the B.E. degree in Electrical and Electronic Engineering from Kyoto University in 2010, and the M.E and $\mathrm{Ph} . \mathrm{D}$. degree in Communications and Computer Engineering, Graduate School of Informatics from Kyoto University, Kyoto, Japan, in 2012 and 2013 respectively. In 2013, he joined the faculty of Communications and Computer Engineering, Graduate School of Informatics, Kyoto University, Japan, where he is currently an Assistant Professor. His current research interests include network design, network management, and wireless communications particularly MAC and TCP design.

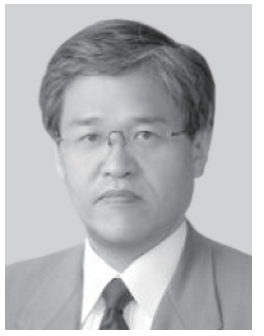

Masahiro Morikura received his B.E. M.E., and Ph.D. degrees in electronics engineering from Kyoto University, Kyoto, Japan in 1979, 1981 and 1991, respectively. He joined NTT in 1981, where he was engaged in the research and development of TDMA equipment for satellite communications. From 1988 to 1989, he was with the Communications Research Centre, Canada, as a guest scientist. From 1997 to 2002, he was active in the standardization of the IEEE 802.11a based wireless LAN. His current research interests include WLANs and M2M wireless systems. He received the Paper Award and the Achievement Award from IEICE in 2000 and 2006, respectively. He also received the Education, Culture, Sports, Science and Technology Minister Award in 2007 and Maejima Award in 2008. Dr. Morikura is now a professor in the Graduate School of Informatics, Kyoto University. He is a member of the IEEE.



Naoki Shinohara received the B.E. degree in electronic engineering, the M.E. and Ph.D. (Eng.) degrees in electrical engineering from Kyoto University, Japan, in 1991, 1993 and 1996, respectively. He was a research associate in the Radio Atmospheric Science Center, Kyoto University from 1998. He was a research associate of the Radio Science Center for Space and Atmosphere, Kyoto University by recognizing the Radio Atmospheric Science Center from 2000, and there he was an associate professor since 2001. He was an associate professor in Research Institute for Sustainable Humanosphere, Kyoto University by recognizing the Radio Science Center for Space and Atmosphere since 2004. From 2010, he has been a professor in Research Institute for Sustainable Humanosphere, Kyoto University. He has been engaged in research on Solar Power Station/Satellite and Microwave Power Transmission system. He is a member of the IEEE, URSI, the Institute of Electronics, Information and Communication Engineers (IEICE) and the Institute of Electrical Engineers of Japan (IEEJ). 